\title{
LINES ON THE FERMAT QUINTIC THREEFOLD AND THE INFINITESIMAL GENERALIZED HODGE CONJECTURE
}

\author{
ALBERTO ALBANO AND SHELDON KATZ
}

\begin{abstract}
We study the deformation theory of lines on the Fermat quintic threefold. We formulate an infinitesimal version of the generalized Hodge conjecture, and use our analysis of lines to prove it in a special case.
\end{abstract}

\section{INTRODUCTION}

A central aspect of the theory of projective algebraic threefolds is the behavior of the curves on them. Although the theory of the period mapping initiated by Griffiths has produced many results, we still lack a general theory. Many problems like the generalized Hodge conjecture are still open, while others have been settled negatively (e.g., the infinite generation of homological modulo algebraic equivalence by Clemens, or the recent counterexample of $\mathrm{C}$. Voisin to the Noether-Lefschetz theorem). It thus seems worthwhile to continue to investigate special cases to gain an understanding of what to expect in general.

In this paper, we consider some questions about rational curves on threefolds with trivial canonical bundle. (The importance of these is clear in the proofs of infinite generation of the Griffiths group of $[\mathrm{Cl} 1, \mathrm{Al}]$, and they play a central role in Reid's program [Re] for the construction of a moduli space for threefolds with trivial canonical bundle). Such a curve $C$ has normal bundle of degree -2 , and so one expects that in the general case $N_{C / X}=\mathscr{O}(-1) \oplus \mathscr{O}(-1)$. This is in fact true for all $C$ of degree $\leq 7$ on a general quintic threefold [K1], but it is not known for other general threefolds with $K \equiv 0$. This is one of the main points in Reid's program. In this case $C$ is isolated in $X$, and by Kodaira's theory [Kod] $C$ deforms with $X$ in all directions, i.e., $C$ is what is called a stable submanifold.

The first part of the paper is dedicated to understanding what happens, from the point of view of deformation theory, when $X$ contains a family of rational curves. We study the case of lines contained in a family $\left\{X_{t}\right\}_{t \in T}$ of smooth quintic hypersurfaces in $\mathbf{P}^{4}$ that contains as special fiber the Fermat quintic

Received by the editors January 16, 1989 and, in revised form, November 20, 1989.

1980 Mathematics Subject Classification (1985 Revision). Primary 14B12, 14C30, 14J30.

The first author was partially supported by M.P.I. funds.

The second author was partially supported by NSF grant RII-8610676. 
threefold, i.e., the quintic $X$ given by the equation

$$
F=x_{0}^{5}+x_{1}^{5}+x_{2}^{5}+x_{3}^{5}+x_{4}^{5}=0
$$

(cf. [K2] for the case when the special fibre is reducible). In $\S 1$ we determine the family of lines on $X$, and in $\S 2$ we determine which lines deform in the family $X_{t}$. While the proofs are computational, we are able to reinterpret our results in term of a geometric object, namely the codifferential $\phi^{*}$ of the Abel-Jacobi mapping $\phi: J(C) \rightarrow J(X)$, where $C$ is the parameter space of the family of lines on $X$. We believe that this interpretation may hold in a more general situation (cf. Problem 2.5).

The second part of the paper deals with the generalized Hodge conjecture. In $\S 3$, we first prove a simple algebraic criterion for an abelian sub-Hodge structure (see Definition 3.1) to deform to first order, and then we formulate an infinitesimal version of the generalized Hodge conjecture. We will refer to this as IGHC (see Conjecture 3.3 for the precise statement). The idea is that, if an abelian sub-Hodge structure $V$ of $H^{3}(X)$ deforms to first order, and we know how to parametrize $V$ with algebraic cycles on $X$, then these cycles should deform to first order with $X$ to parametrize the deformation of $V$.

We again study this question for $X=$ Fermat quintic threefold. The generalized Hodge conjecture is true for $X$ ( $\mathrm{cf}$. [Ra, S1]), and we prove IGHC for the deformations of some natural sub-Hodge structures of $H^{3}(X)$. It turns out that the algebraic conditions for $V$ to deform, when properly formulated in the case at hand, are exactly the ones giving the deformation of the algebraic cycles parametrizing $V$, thus connecting this problem with the deformation theory of submanifolds, and giving evidence for IGHC in general.

It is hoped that the study of IGHC will provide a useful tool for investigating the generalized Hodge conjecture for threefolds near a given one for which one knows that the generalized Hodge conjecture is true. As a trivial case, Griffiths' result that the generalized Hodge conjecture holds for a generic quintic threefold is obtainable by producing an example with no nontrivial abelian sub-Hodge structure and then deforming it. It would be nice to use this technique to find examples of families of threefolds for which the generalized Hodge conjecture is true, yet the general member of the family has a nontrivial abelian sub-Hodge structure.

Unfortunately, our proofs rely on the explicit decomposition of $H^{3}(X)$ into eigenspaces of the action of (part of) $\operatorname{Aut}(X)$ (given by Ogus in [O]), and so they do not suggest a general method of attack. Nevertheless, we think that it would be interesting to better understand the relationship between deformations of abelian sub-Hodge structures and deformations of algebraic cycles.

Finally, we want to draw attention to the recent theory of deformations of submanifolds via $\mathscr{D}$-modules, initiated by Clemens (cf. [Cl2]). Though we make no use of it, it should prove a useful tool in investigating more general situations than the one we treat here. 
We would like to express our gratitude to $\mathrm{H}$. Clemens for raising questions about the deformations of lines on the Fermat quintic that led to the present paper. We are grateful to C. Schoen for a series of lengthy correspondences with the second named author which generated many ideas related to the Fermat quintic threefold. We also want to thank R. Smith and R. Varley for useful conversations, and A. Collino for suggesting that a direct computation would have been the easiest way to determine the family of lines on a Fermat threefold. The first named author wants to thank the Department of Mathematics at Oklahoma State University for the support given to him during the preparation of this paper, as well as for the friendly atmosphere and stimulating working environment.

Convention. We work exclusively over the field $\mathbf{C}$ of complex numbers.

\section{Lines ON Fermat threEFoldS}

Let $X$ be the hypersurface in $\mathbf{P}^{4}$ given by the equation $x_{0}^{n}+x_{1}^{x}+x_{2}^{n}+x_{3}^{n}+$ $x_{4}^{n}=0$. We will call $X$ a Fermat threefold. Let now $\zeta$ be a complex number such that $\zeta^{n}=1$. Then the hyperplanes $x_{i}+\zeta^{r} x_{j}=0$ intersect $X$ in a cone of degree $n$, so that there are $10 n$ cones on $X$. The purpose of this section is to show that if $n \geq 4$, then the only lines on $X$ are the ones that lie in one of the cones. We first prove an analogous property for the Fermat surfaces.

Proposition 1.1. Let $S$ be the surface in $\mathbf{P}^{3}$ given by the equation $x_{0}^{n}+x_{1}^{n}+$ $x_{2}^{n}+x_{3}^{n}=0, n \geq 3$. If $l$ is a line contained in $S$, then $l$ goes through a point $\left(\alpha_{0}: \alpha_{1}: \alpha_{2}: \alpha_{3}\right)$ with 2 coordinates equal to 0 .

Proof. Let $\alpha=\left(\alpha_{0}: \alpha_{1}: \alpha_{2}: \alpha_{3}\right), \beta=\left(\beta_{0}: \beta_{1}: \beta_{2}: \beta_{3}\right) \in S$ be two points on $l$. We can normalize the coordinates so that $\alpha=\left(0: \alpha_{1}: \alpha_{2}: \alpha_{3}\right)$ and $\beta=\left(\beta_{0}: \beta_{1}: \beta_{2}: 0\right)$. Let us assume that $\alpha_{1} \alpha_{2} \alpha_{3} \neq 0$. Then it is enough to show that $\beta_{0} \beta_{1} \beta_{2}=0$. The parametric equation of $l$ is: $P=\lambda \alpha+\mu \beta$. Substituting in the equation of $S$ and imposing that it be identically satisfied in $\lambda, \mu$, we obtain the following system of equations:

$$
\left\{\begin{array}{c}
\alpha_{1} \beta_{1}^{n-1}+\alpha_{2} \beta_{2}^{n-1}=0 \\
\vdots \\
\alpha_{1}^{n-1} \beta_{1}+\alpha_{2}^{n-1} \beta_{2}=0
\end{array}\right.
$$

If $\beta_{1}=0$ or $\beta_{2}=0$, we are done. If not, then $\alpha_{1}=-\alpha_{2}\left(\beta_{2} / \beta_{1}\right)^{n-1}$, and $\alpha_{1}^{2}=$ $-\alpha_{2}^{2}\left(\beta_{2} / \beta_{1}\right)^{n-2}$. Substituting and dividing by $\alpha_{2}^{2} \beta_{2}^{n-2}$, we get $\left(\beta_{2} / \beta_{1}\right)^{n}=-1$, and so $\beta_{1}^{n}+\beta_{2}^{n}=0$. But $\beta \in S$, so this implies $\beta_{0}=0$. Q.E.D.

We consider now the three-fold case.

Proposition 1.2. Let $X$ be the hypersurface in $\mathbf{P}^{4}$ given by the equation $x_{0}^{n}+$ $x_{1}^{n}+x_{2}^{n}+x_{3}^{n}+x_{4}^{n}=0, n \geq 3$. Let $\alpha=\left(\alpha_{0}: \alpha_{1}: 0: 0: 0\right)$ and let $\alpha \in l \subset X$. Then $l$ belongs to one of the cones. 
Proof. Let $\beta=\left(\beta_{0}: \beta_{1}: \beta_{2}: \beta_{3}: \beta_{4}\right) \in l$. As before, the parametric equation of $l$ is: $P=\lambda \alpha+\mu \beta$. Therefore we obtain the following system:

$$
\left\{\begin{array}{c}
\alpha_{0} \beta_{0}^{n-1}+\alpha_{1} \beta_{1}^{n-1}=0 \\
\vdots \\
\alpha_{0}^{n-1} \beta_{0}+\alpha_{1}^{n-1} \beta_{1}=0
\end{array}\right.
$$

Then, as before, we obtain that $\beta_{0}^{n}+\beta_{1}^{n}=0$, and since $\beta \in X$, we have that $\beta_{2}^{n}+\beta_{3}^{n}+\beta_{4}^{n}=0$, i.e. $l \cap\left\{x_{0}=x_{1}=0\right\}$ is on a plane Fermat curve. Q.E.D.

We are ready now to prove

Proposition 1.3. Let $X$ be the hypersurface in $\mathbf{P}^{4}$ given by the equation $x_{0}^{n}+$ $x_{1}^{n}+x_{2}^{n}+x_{3}^{n}+x_{4}^{n}=0$. Assume $n \geq 4$. If $l$ is a line contained in $X$, then $l$ goes through a point $\left(\alpha_{0}: \alpha_{1}: \alpha_{2}: \alpha_{3}: \alpha_{4}\right)$ with 3 coordinates equal to 0 .

Proof. Let $\alpha=\left(\alpha_{0}: \alpha_{1}: \alpha_{2}: \alpha_{3}: \alpha_{4}\right), \beta=\left(\beta_{0}: \beta_{1}: \beta_{2}: \beta_{3}: \beta_{4}\right) \in X$ be two points on $l$. We can normalize the coordinates so that $\alpha=\left(0: \alpha_{1}: \alpha_{2}: \alpha_{3}: \alpha_{4}\right)$ and $\beta=\left(\beta_{0}: \beta_{1}: \beta_{2}: \beta_{3}: 0\right)$. If $\alpha_{4} \beta_{0}=0$, then we are done by Proposition 1.1 , so assume that $\alpha_{4} \beta_{0} \neq 0$. This time, the system that we have to solve is

$$
\left\{\begin{array}{c}
\alpha_{1} \beta_{1}^{n-1}+\alpha_{2} \beta_{2}^{n-1}+\alpha_{3} \beta_{3}^{n-1}=0 \\
\alpha_{1}^{2} \beta_{1}^{n-2}+\alpha_{2}^{2} \beta_{2}^{n-2}+\alpha_{3}^{2} \beta_{3}^{n-2}=0 \\
\alpha_{1}^{3} \beta_{1}^{n-3}+\alpha_{2}^{3} \beta_{2}^{n-3}+\alpha_{3}^{3} \beta_{3}^{n-3}=0 \\
\vdots \\
\alpha_{1}^{n-1} \beta_{1}+\alpha_{2}^{n-1} \beta_{2}+\alpha_{3}^{n-1} \beta_{3}=0
\end{array}\right.
$$

and we note that, since $n \geq 4$, the first three equations are distinct.

Let us assume first that $\alpha_{1}=0$. Then, if $\alpha_{2}=0$ we are done. If not, from the last equation we can write

$$
\beta_{2}=-\beta_{3}\left(\frac{\alpha_{3}}{\alpha_{2}}\right)^{n-1}
$$

and substituting in the next to last, we obtain

$$
\alpha_{2}^{n-2} \beta_{3}^{2}\left(\frac{\alpha_{3}}{\alpha_{2}}\right)^{2 n-2}=-\alpha_{3}^{n-2} \beta_{3}^{2} .
$$

If $\beta_{3}=0$, then $\alpha_{2} \beta_{2}=0$, so we are done. If $\alpha_{3}=0$, we are done. If not, we can divide by $\beta_{3}^{2} \alpha_{3}^{n-2}$ and we obtain, as before

$$
\alpha_{2}^{n}+\alpha_{3}^{n}=0 \text {. }
$$

Recalling that $\alpha \in X$ and that $\alpha_{0}=\alpha_{1}=0$, this implies that $\alpha_{4}=0$, so we are done. 
We can now assume that $\alpha_{1} \alpha_{2} \alpha_{3} \beta_{1} \beta_{2} \beta_{3} \neq 0$. Then, solving the first three equations for $\alpha_{1}, \alpha_{1}^{2}$ and $\alpha_{1}^{3}$ respectively, we have the (obvious) relation: $\alpha_{1} \alpha_{1}^{3}=\left(\alpha_{1}^{2}\right)^{2}$. Writing this explicitly, we get

$$
\begin{gathered}
\alpha_{2}^{4}\left(\frac{\beta_{2}}{\beta_{1}}\right)^{2 n-4}+\alpha_{2} \alpha_{3}^{3} \frac{\beta_{2}^{n-1} \beta_{3}^{n-3}}{\beta_{1}^{2 n-4}}+\alpha_{2}^{3} \alpha_{3} \frac{\beta_{2}^{n-3} \beta_{3}^{n-1}}{\beta_{1}^{2 n-4}}+\alpha_{3}^{4}\left(\frac{\beta_{3}}{\beta_{1}}\right)^{2 n-4} \\
=\alpha_{2}^{4}\left(\frac{\beta_{2}}{\beta_{1}}\right)^{2 n-4}+2 \alpha_{2}^{2} \alpha_{3}^{2} \frac{\beta_{2}^{n-2} \beta_{3}^{n-2}}{\beta_{1}^{2 n-4}}+\alpha_{3}^{4}\left(\frac{\beta_{3}}{\beta_{1}}\right)^{2 n-4} .
\end{gathered}
$$

Canceling and clearing denominators, we obtain

$$
2 \alpha_{2}^{2} \alpha_{3}^{2} \beta_{2}^{n-2} \beta_{3}^{n-2}=\alpha_{2} \alpha_{3}^{3} \beta_{2}^{n-1} \beta_{3}^{n-3}+\alpha_{2}^{3} \alpha_{3} \beta_{2}^{n-3} \beta_{3}^{n-1} .
$$

Finally, dividing by $\alpha_{2} \alpha_{3} \beta_{2}^{n-3} \beta_{3}^{n-3}$ gives

$$
2 \alpha_{2} \alpha_{3} \beta_{2} \beta_{3}=\left(\alpha_{3} \beta_{2}\right)^{2}+\left(\alpha_{2} \beta_{3}\right)^{2},
$$

i.e., $\left[\alpha_{3} \beta_{2}-\alpha_{2} \beta_{3}\right]^{2}=0$, so that

$$
\alpha_{3} \beta_{2}=\alpha_{2} \beta_{3} .
$$

Similarly, we obtain that $\alpha_{3} \beta_{1}=\alpha_{1} \beta_{3}$, and $\alpha_{2} \beta_{1}=\alpha_{1} \beta_{2}$, and hence $\alpha_{i}=$ $\lambda \beta_{i}$, for $i=1,2,3$. But then we have, from any of the equations (3), that $\alpha_{1}^{n}+\alpha_{2}^{n}+\alpha_{3}^{n}=0$, and since $\alpha \in X$ and $\alpha_{0}=0$, this implies $\alpha_{4}=0$, contrary to the assumption. So $\alpha_{1} \alpha_{2} \alpha_{3} \beta_{1} \beta_{2} \beta_{3}=0$, and we are done. Q.E.D.

\section{Deformation THEORY OF THE LINES ON THE FERMAT QUINTIC THREEFOLD}

Let $X$ be a quintic threefold, i.e., a hypersurface of degree 5 in $\mathbf{P}^{4}$. It is well known $[\mathrm{H}]$ that the generic such $X$ contains exactly 2875 distinct lines. On the other hand, we have seen that on the Fermat quintic there are 50 1-dimensional families of lines. If $\left\{X_{t}\right\}_{t \in T}$ is a family of quintics, with $X_{0}=$ Fermat quintic, then we expect that only finitely many lines of $X_{0}$ will move with $X_{t}$. So the problem we are interested in is the following: let $p: \mathscr{X} \rightarrow T$ be a deformation of $X_{0}=p^{-1}(0)=$ Fermat quintic. Then for certain lines $l \subset X_{0}$ there will exist a subvariety $\mathscr{L} \subset \mathscr{X}$ such that $p_{\mid \mathscr{L}}^{-1}(0)=n l, n \geq 1$. We want to identify these lines.

What we are studying is the problem of deformations of the pair $(l, X)$. There is a general theory for this, for first order deformations, i.e., deformations parametrized by $\Delta=\operatorname{Spec} D$, where $D=\mathbf{C}[\varepsilon] / \varepsilon^{2}$ is the ring of dual numbers (cfr. [Kod]), of which we give here only a brief account. Consider the complex of coherent sheaves on $X$

$$
\Theta_{X} \rightarrow N_{l / X} \rightarrow 0
$$


where $\Theta_{X}$ is the tangent bundle of $X, N_{l / X}$ is the normal bundle of $l$ in $X$ and we consider $N_{l / X}$ as a sheaf on $X$ extending by zero. Then the first hypercohomology group $\mathscr{H}^{1}$ of the complex fits into the exact sequence

$$
H^{0}\left(N_{l / X}\right) \rightarrow \mathscr{H}^{1} \rightarrow H^{1}\left(\Theta_{X}\right) \rightarrow H^{1}\left(N_{l / X}\right) .
$$

The central fact is that $\mathscr{H}^{1}$ is the tangent space to the deformation space of the pair $(l, X)$, while $H^{1}\left(\Theta_{X}\right)$ is the tangent space to the deformation space of $X$ and so $l$ moves (to first order) with $X$ if and only if the (tangent) direction in which $X$ moves is in the image of $\mathscr{H}^{1}$.

Let now $X=\{F=0\}=\left\{x_{0}^{5}+x_{1}^{5}+x_{2}^{5}+x_{3}^{5}+x_{4}^{5}=0\right\}$ be the Fermat quintic threefold. For $\rho \in H^{1}\left(\Theta_{X}\right)$, let $X_{\rho} \rightarrow \Delta$ be the corresponding first order deformation. By Griffiths' theory [Gr1], $H^{1}\left(\Theta_{X}\right) \cong H^{0}\left(\mathscr{O}_{\mathbf{P}^{4}}(5)\right) /\left(F_{x_{0}}, \ldots, F_{x_{4}}\right)$, so that $X_{\rho}=\{F+t G=0\}$, with $G$ a polynomial of degree 5 .

Proposition 2.1. For generic $\rho \in H^{1}\left(\Theta_{X}\right)$, no line $l$ moves in the direction of $\rho$, i.e., all pairs $(l, X)$ are obstructed to first order.

Proof. Up to the action of the automorphism group of $X$, we can write the parametric equations of $l$ as $l=(u:-u: a v: b v: c v)$, where $(u, v)$ are homogeneous coordinates on $\mathbf{P}^{1}$, and $a, b, c \in \mathbf{C}$ are such that $a^{5}+b^{5}+c^{5}=$ 0 . Hence a deformation $l_{t}$ of $l=l_{0}$ has parametric equations

$$
l_{t}=\left(u+t \alpha_{0}:-u+t \alpha_{1}: a v+t \alpha_{2}: b v+t \alpha_{3}: c v+t \alpha_{4}\right),
$$

where the $\alpha_{i}$ 's are linear forms in $(u, v)$, and $t^{2}=0$.

Since the family $X_{\rho}$ is given by $X_{t}=\{F+t G=0\}$, for $l_{t} \subset X_{t}$, we must have $(F+t G)_{\mid l_{t}} \equiv 0$.

But $F_{\mid l}=u^{5}+5 u^{4} \alpha_{0} t-u^{5}+5 u^{4} \alpha_{1} t+a^{5} v^{5}+5 a^{4} v^{4} \alpha_{2} t+b^{5} v^{5}+5 b^{4} v^{4} \alpha_{3} t+$ $c^{5} v^{5}+5 c^{4} v^{4} \alpha_{4} t=t\left(u^{4} \alpha+v^{4} \beta\right)$, for some $\alpha, \beta$ linear forms in $(u, v)$, and hence the coefficients of the monomials $u^{3} v^{2}$ and $u^{2} v^{3}$ in $G_{\mid l_{0}}$ must be zero. These coefficients are homogeneous polynomials in $(a, b, c)$ of degree 2 and 3 respectively so, for generic $\mathrm{G}$, these two equations have no common solution on the curve $a^{5}+b^{5}+c^{5}=0$ and hence $l_{0}$ does not deform in the direction of $G$. Q.E.D.

Remark. Note that $H^{1}\left(N_{l / X}\right)=H^{1}(\mathscr{O}(1) \oplus \mathscr{O}(-3))$ is two-dimensional, so we expect two conditions for the pair to deform.

We study now what happens when we allow deformations with monodromy, i.e., we consider deformations of the type $X_{\rho}=\left\{F+t^{n} G=0\right\}$, with $n \geq 2$.

Proposition 2.2. For generic $\rho \in H^{1}\left(\Theta_{X}\right)$, there are exactly 10 lines on each cone that deform (to second order) in the direction of $\rho$ with monodromy $\mathbf{Z}_{2}$.

Proof. The proof is similar to that of Proposition 2.1. We write the parametric equations of $l$ as $l=(u:-u: a v: b v: c v)$, where $(u, v)$ are homogeneous 
coordinates on $\mathbf{P}^{1}$, and $a, b, c \in \mathbf{C}$ are such that $a^{5}+b^{5}+c^{5}=0$. A second order deformation $l_{t}$ of $l=l_{0}$ has parametric equations:

$$
\begin{gathered}
l_{t}=\left(u+t \alpha_{0}^{\prime}+t^{2} \alpha_{0}^{\prime \prime}:-u+t \alpha_{1}^{\prime}+t^{2} \alpha_{1}^{\prime \prime}: a v+t \alpha_{2}^{\prime}+t^{2} \alpha_{2}^{\prime \prime}:\right. \\
\left.b v+t \alpha_{3}^{\prime}+t^{2} \alpha_{3}^{\prime \prime}: c v+t \alpha_{4}^{\prime}+t^{2} \alpha_{4}^{\prime \prime}\right),
\end{gathered}
$$

where the $\alpha_{i}^{\prime}$ 's, $\alpha_{i}^{\prime \prime}$ 's are linear forms in $(u, v)$ and $t^{3}=0$.

The family $X_{\rho}$ is now given by $X_{t}=\left\{F+t^{2} G=0\right\}$, and for $l_{t}$ to be contained in $X_{t}$, we must have $\left(F+t^{2} G\right)_{\mid I_{t}} \equiv 0$.

Substituting,

$$
\begin{aligned}
F_{\mid l_{t}}= & 5 t\left(u^{4} \alpha_{0}^{\prime}+u^{4} \alpha_{1}^{\prime}+a^{4} v^{4} \alpha_{2}^{\prime}+b^{4} v^{4} \alpha_{3}^{\prime}+c^{4} v^{4} \alpha_{4}^{\prime}\right) \\
& +5 t^{2}\left(2 u^{3} \alpha_{0}^{\prime 2}+u^{4} \alpha_{0}^{\prime \prime}-2 u^{3} \alpha_{1}^{\prime 2}+u^{4} \alpha_{1}^{\prime \prime}+2 a^{3} v^{3} \alpha_{2}^{\prime 2}\right. \\
& \left.\quad+a^{4} v^{4} \alpha_{2}^{\prime \prime}+2 b^{3} v^{3} \alpha_{3}^{\prime 2}+b^{4} v^{4} \alpha_{3}^{\prime \prime}+2 c^{3} v^{3} \alpha_{4}^{\prime 2}+c^{4} v^{4} \alpha_{4}^{\prime \prime}\right) \\
= & 5 t\left(u^{4} A+v^{4} B\right)+5 t^{2}\left(u^{3} C+v^{3} D\right)
\end{aligned}
$$

and so we must have

$$
u^{4} A+v^{4} B=0, \quad u^{3} C+v^{3} D=-G_{\left.\right|_{0}},
$$

where $A, B$ are linear and $C, D$ are quadratic. We now can choose $\alpha_{0}^{\prime}=-\alpha_{1}^{\prime}$, and $\alpha_{2}^{\prime}=b^{4} \delta+c^{4} \gamma, \alpha_{3}^{\prime}=-a^{4} \delta+c^{4} \varepsilon, \alpha_{4}^{\prime}=-a^{4} \gamma-b^{4} \varepsilon$, for any $\delta, \gamma, \varepsilon$ linear forms. For later reference, we note that this gives a 6-dimensional vector space of solutions for the $\alpha_{i}^{\prime}$. With these choices, the first equation is satisfied and, substituting in the second one we find

$$
\begin{aligned}
u^{3} C+v^{3} D= & u^{4}\left(\alpha_{0}^{\prime \prime}+\alpha_{1}^{\prime \prime}\right)+v^{4}\left(a^{4} \alpha_{2}^{\prime \prime}+b^{4} \alpha_{3}^{\prime \prime}+c^{4} \alpha_{4}^{\prime \prime}\right) \\
& +2 v^{3}\left(a^{3} b^{3}\left(a^{5}+b^{5}\right) \delta^{2}+a^{3} c^{3}\left(a^{5}+c^{5}\right) \gamma^{2}+b^{3} c^{3}\left(b^{5}+c^{5}\right) \varepsilon^{2}\right. \\
& \left.+2 a^{3} b^{3} c^{3}(b c \delta \gamma-a c \delta \varepsilon+a b \gamma \varepsilon)\right) .
\end{aligned}
$$

So the coefficient of $u^{3} v^{2}$ in $G_{\mid l_{0}}$ must be zero, but if $a b c \neq 0$, then the coefficient of $u^{2} v^{3}$ in $v^{3} D$ is nonzero and we can then find $\gamma, \delta, \varepsilon, \alpha_{i}^{\prime \prime}$ such that the second equation is satisfied. As we remarked before, the coefficient of $u^{3} v^{2}$ is a quadratic polynomial $Q(a, b, c)$, and so the conclusion is: given $G$, a line $l=(u:-u: a v: b v: c v)$ moves in the direction of $G$ with monodromy $\mathbf{Z}_{2}$ only if $Q(a, b, c)=a^{5}+b^{5}+c^{5}=0$. If, on the other hand, $a b c=0$, then for all choices of $\alpha_{i}^{\prime}, \alpha_{i}^{\prime \prime}$ in the deformation of $l_{0}$ we have that $u^{3} C+v^{3} D$ does not contain the monomials $u^{3} v^{2}$ and $u^{2} v^{3}$, so that the lines where $a$ or $b$ or $c$ is 0 do not deform with monodromy $\mathbf{Z}_{2}$. Q.E.D.

In exactly the same way, we can prove the following proposition. 
Proposition 2.3. For generic $\rho \in H^{1}\left(\Theta_{X}\right)$, the lines that lie on more than one cone deform in the direction of $\rho$ with monodromy $\mathbf{Z}_{5}$.

Proof. Just as before, look at a fifth order deformation of $l_{0}=(u:-u: a v: b v$ : 0 ) in the family $X_{t}=\left\{F+t^{5} G=0\right\}$, with $t^{6}=0$. We write the deformation of $l_{0}$ as

$$
\begin{gathered}
l_{t}=\left(u+t \alpha_{0}^{\prime}+t^{2} \alpha_{0}^{\prime \prime}+t^{3} \alpha_{0}^{\prime \prime \prime}+t^{4} \alpha_{0}^{(i v)}+t^{5} \alpha_{0}^{(v)}:\right. \\
-u+t \alpha_{1}^{\prime}+t^{2} \alpha_{1}^{\prime \prime}+t^{3} \alpha_{1}^{\prime \prime \prime}+t^{4} \alpha_{1}^{(i v)}+t^{5} \alpha_{1}^{(v)}: \\
a v+t \alpha_{2}^{\prime}+t^{2} \alpha_{2}^{\prime \prime}+t^{3} \alpha_{2}^{\prime \prime \prime}+t^{4} \alpha_{2}^{(i v)}+t^{5} \alpha_{2}^{(v)}: \\
b v+t \alpha_{3}^{\prime}+t^{2} \alpha_{3}^{\prime \prime}+t^{3} \alpha_{3}^{\prime \prime \prime}+t^{4} \alpha_{3}^{(i v)}+t^{5} \alpha_{3}^{(v)}: \\
\left.t \alpha_{4}^{\prime}+t^{2} \alpha_{4}^{\prime \prime}+t^{3} \alpha_{4}^{\prime \prime \prime}+t^{4} \alpha_{4}^{(i v)}+t^{5} \alpha_{4}^{(v)}\right) .
\end{gathered}
$$

Then $F_{\mid l_{t}}=t A_{1}+t^{2} A_{2}+t^{3} A_{3}+t^{4} A_{4}+t^{5} A_{5}$ and so we must have $A_{i}=0$ for $i=1, \ldots, 4$, and $A_{5}=-G_{\mid l_{0}}$. If we choose $\alpha_{0}^{j}=-\alpha_{1}^{j}, \alpha_{2}^{j}=-(b / a)^{4} \alpha_{3}^{j}$, for $j=1, \ldots, 4$, then a simple substitution shows that $A_{i}=0$ for $i \leq 4$, and $A_{5}=u^{4}\left(\alpha_{0}^{(v)}-\alpha_{1}^{(v)}\right)+v^{4}\left(a^{4} \alpha_{2}^{(v)}+b^{4} \alpha_{3}^{(v)}\right)+\alpha_{4}^{\prime 5}$. Since $\alpha_{4}^{\prime}=\lambda u+\mu v$, we see that the monomials $u^{3} v^{2}$ and $u^{2} v^{3}$ have coefficients $10 \lambda^{3} \mu^{2}, 10 \lambda^{2} \mu^{3}$ respectively in $A_{5}$. Hence, if the coefficients of these monomials in $G_{\mid l_{0}}$ are both nonzero, which is true for $G$ generic, we can solve for $\lambda, \mu$, and then choose $\alpha_{0}^{(v)}, \ldots, \alpha_{3}^{(v)}$ appropriately, to satisfy the last condition. Hence $l_{0}$ deform in the direction of $G$ with $\mathbf{Z}_{5}$ monodromy. Q.E.D.

Remarks. 1. Let $\left\{X_{t}\right\}_{t \in T}$ be a family of quintics, with $X_{0}=$ Fermat quintic, and assume that on $X_{t}$ for $t \neq 0$ there are only finitely many lines. Let $\mathscr{L}=\left\{(l, t) \in \operatorname{Gr}(2,5) \times T \mid l \subset X_{t}\right\}$ be the incidence correspondence. Then the projection $\pi: \mathscr{L} \rightarrow T$ is a generically finite map of degree 2875 , and is ramified at $t=0$. By Fulton's theory [F], there is a 0 -cycle of degree 2875 on the distinguished components of $\mathscr{L}_{0}=\mathscr{L} \cdot \pi^{-1}(0)$ well determined up to rational equivalence. Propositions 2.2 and 2.3 determine a cycle representing this class: it is given by the class of a canonical divisor on each of the 50 curves parametrizing the lines on the cones, counted with multiplicity 2 , and the 375 points corresponding to the lines that lie in two cones, each counted with multiplicity 5 . We have: $(50 \times 10 \times 2)+(375 \times 5)=2875$. To see this, let $Q$ be the universal quotient bundle on $\operatorname{Gr}(2,5)$, and apply [F, Corollary 11.1] to the diagram [F, p. 197]

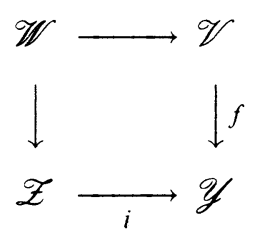

where $\mathscr{Y}=\operatorname{Sym}^{5}(Q), i: \mathscr{Z} \rightarrow \mathscr{Y}$ is the embedding of the 0 -section and $f: \mathscr{V} \rightarrow \mathscr{Y}$ is the embedding of the section of $\operatorname{Sym}^{5}(Q)$ given by an equation 
for $\left\{X_{t}\right\}_{t \in T}$ (note the slight change in notation from $\left.[\mathrm{F}]\right)$. Since $\mathscr{Z}_{t}$ meets $\mathscr{V}_{t}$ properly for $t \neq 0$ by assumption, we can apply the paragraph after Corollary 11.1 of $[\mathrm{F}]$ to conclude that our cycle coincides with Fulton's limit intersection cycle.

2. Experience has shown that determining the multiplicities as in Propositions $2.2,2.3$ is often the most difficult part of a deformation theory analysis. What is still needed is a direct method to a priori determine these multiplicities with less calculations.

Proposition 2.4. For the generic deformation $X_{t}=F+t G+t^{2} H+\cdots=0$ of the Fermat quintic threefold, the lines described in Propositions 2.2 and 2.3 are precisely the limits of the 2875 lines on $X_{t}$.

Proof. We will only prove this for the lines of Proposition 2.2. The proof is similar in the other case.

As in the proof of Proposition 2.2, we introduce $Z_{2}$ monodromy, i.e. we rewrite the equation as $F+t^{2} G+t^{4} H+\cdots=0$. We write

$$
\begin{aligned}
& l_{t}=\left(u+\sum_{n} \alpha_{0}^{(n)} t^{n},-u+\sum_{n} \alpha_{1}^{(n)} t^{n}, a v\right.+\sum_{n} \alpha_{2}^{(n)} t^{n}, \\
&\left.b v+\sum_{n} \alpha_{3}^{(n)} t^{n}, c v+\sum_{n} \alpha_{4}^{(n)} t^{n}\right)
\end{aligned}
$$

and inductively solve for the $\alpha_{i}^{(n)}$. We show that there is a four-dimensional family of solutions, reflecting the action of $\mathrm{GL}(2)$ on $\mathbf{P}^{1}$ which gives a 4dimensional family of parameterizations for any line. We know that for the 10 lines of Proposition 2.2, we can solve for the $\alpha_{i}^{\prime}, \alpha_{i}^{\prime \prime}$ to begin the induction.

The detailed computation being a little messy, we merely summarize how to carry out the induction. The interested reader can consult [K3, Lemma 2.7] to see more details in a similar computation.

The main point is to refine the inductive hypothesis to say that for all $n$, one can solve for a family of solutions for $\alpha_{i}^{\prime}, \ldots, \alpha_{i}^{(n)}$ in such a way that the following are true:

i. $\left.\left(F+t^{2} G+t^{4} H+\cdots\right)\right|_{l_{t}}=0$ modulo $t^{n+1}$.

ii. The $\alpha_{i}^{\prime}, \ldots, \alpha_{i}^{(n-3)}$ found in the preceding step are not altered.

iii. A nontrivial linear condition is imposed on the family of solutions for the $\alpha_{i}^{(n-2)}$, giving a 4-dimensional solution space.

iv. A nontrivial linear condition is imposed on the family of solutions for the $\alpha_{i}^{(n-1)}$, giving a 5-dimensional solution space.

v. There is a 6-dimensional family of solutions for the $\alpha_{i}^{(n)}$. The linear form $\alpha_{0}^{(n)}+\alpha_{1}^{(n)}$ is constant in this family.

The proof proceeds by computing i. This expression has the following properties. 
a. The $\alpha_{i}^{(n)}$ appear in the coefficient of $t^{n}$ as the expression $u^{4}\left(\alpha_{0}^{(n)}+\alpha_{1}^{(n)}\right)+v^{4}\left(a^{4} \alpha_{2}^{(n)}+b^{4} \alpha_{3}^{(n)}+c^{4} \alpha_{4}^{(n)}\right.$ ) (see proof of Proposition 2.2 for an example of this).

b. The terms $\alpha_{i}^{(n-1)}$ appear linearly in the coefficient of $t^{n}$ in such a way that the only occurrence of the $\alpha_{i}^{(n-1)}$ in a multiple of $u^{3} t^{n}$ is in the term $u^{3} \alpha_{1}^{\prime}\left(\alpha_{0}^{(n-1)}+\alpha_{1}^{(n-1)}\right)$.

c. Once $n \geq 5$, the terms $\alpha_{i}^{(n-2)}$ occur linearly.

d. The higher derivatives $H, \ldots$ of the deformation do not affect the coefficients of the $\alpha_{i}^{(k)}$ for $k=n-2, n-1, n$.

The cases $n \leq 2$ are done. $n=3,4$ can be done with minor modifications of the general case. (It is necessary to compute using the expressions $\gamma, \delta, \varepsilon$ appearing in the proof of Proposition 2.2. The point is that these will occur in fairly simple symmetric form as in the proof of Proposition 2.2, so they will be easy to compute with). From now on, assume that $n \geq 5$, so that a-d hold. Assume inductively that we have found the first $n-1$ derivatives. We must solve for the $\alpha_{i}^{(k)}, k=n-2, n-1, n$.

The coefficient of $u^{3} v^{2} t^{n}$ gives a linear condition on the $\alpha_{i}^{(k)}$, by a, b, and c. By a, the $\alpha_{i}^{(n)}$ do not occur, and by b combined with $\mathrm{v}$ and the inductive hypothesis, changing the $\alpha_{i}^{(n-1)}$ do not affect this expression. So this condition is imposed on the space of solutions for the $\alpha_{i}^{(n-2)}$. It is easy to see that for general deformations, this linear condition is independent of the previous conditions, reducing the solution space from 5-dimensional to 4-dimensional. (In fact, by $\mathrm{d}$ this is actually true for general $G$ which is consistent with the findings of Propostions 2.2 and 2.3). This proves iii.

The coefficient of $u^{2} v^{3} t^{n}$ now gives a linear condition on the $\alpha_{i}^{(k)}$ by a, b, and c. We now need only be interested in $k=n-1, n$. By a, the $\alpha_{i}^{(n)}$ do not occur. Hence we get a linear condition on the $\alpha_{i}^{(n-1)}$ which is easily seen to always be independent of the previous conditions. This reduces the dimension of the solution space for the $\alpha_{i}^{(n-1)}$ from 6 to 5 , proving iv.

Finally, we look at the remaining terms in the coefficient of $t^{n}$ (the terms in the ideal $\left.\left(u^{4}, v^{4}\right)\right)$. By a, the resulting system of equations on the $\alpha_{i}^{(n)}$ is of the form $u^{4} A+v^{4} B=$ constant, where $A, B$ are precisely as in the proof of Proposition 2.2, with the $\alpha_{i}^{\prime}$ replaced by $\alpha_{i}^{(n)}$. This equation has a 6-dimensional space of solutions (a translate of the vector space of solutions found in the earlier proof). Since $A$ is $\alpha_{0}^{(n)}+\alpha_{1}^{(n)}$, v is proved.

The proof concludes by invoking Artin's approximation theorem [Ar], and noting that since we have already accounted for 2875 lines, there can be no others. Q.E.D.

Going back to the proof of Proposition 2.2, we constructed a map $\psi$ : $H^{1}\left(X, \Theta_{X}\right) \rightarrow H^{0}\left(C, K_{C}\right)$, where $C$ is the plane quintic $x_{2}^{5}+x_{3}^{5}+x_{4}^{5}=0$ in the plane $x_{0}=x_{1}=0$, by associating to a polynomial $G$ of degree 5 the 
conic $Q\left(x_{2}, x_{3}, x_{4}\right)$ that is the coefficient of $u^{3} v^{2}$ in the restriction of $G$ to $l$. There is another natural map $H^{1}\left(X, \Theta_{X}\right) \rightarrow H^{0}\left(C, K_{C}\right)$, namely the codifferential $\phi^{*}$ of the Abel-Jacobi mapping $\phi: J(C) \rightarrow J(X)$, where $J(X)$ is the intermediate Jacobian of $X$. We want now to show that these maps are the same. For this purpose, we have to describe the Hodge structures of $X$ and $C$.

Let $X$ be the Fermat quintic threefold, with equation $F=x_{0}^{5}+\cdots+x_{4}^{5}=0$. The following facts may be found in [O, S1].

Let $G=\mathbf{Z}_{5}^{5} / \Delta$ act on $X$ by

$$
\left(a_{0}, \ldots, a_{4}\right) \cdot\left(x_{0}, \ldots, x_{4}\right)=\left(\zeta^{a_{0}} x_{0}, \ldots, \zeta^{a_{4}} x_{4}\right),
$$

where $\zeta=e^{2 \pi i / 5}$, and $\Delta$ is the diagonal. The group of characters of $G$ is

$$
\hat{G}=\left\{\left(a_{0}, \ldots, a_{4}\right) \in \mathbf{Z}_{5}^{5} \mid \sum_{i} a_{i} \equiv 0(\bmod 5)\right\} .
$$

The eigenspaces of the $G$-action on $H^{3}(X, \mathrm{C})$ are one dimensional; the characters which occur are $\left\{\left(a_{0}, \ldots, a_{4}\right) \in \hat{G} \mid \forall i a_{i} \neq 0\right\}$. The characters occurring in $H^{3-q, q}$ are those for which $\sum_{i} a_{i}=5(q+1)$, thinking of the $a_{i}$ as integers between 1 and 4 . In fact, these are eigenspaces for $H^{3}(X, \mathbf{Q}(\zeta))$, and the action of $\operatorname{Gal}(\mathbf{Q}(\zeta) / \mathbf{Q})$ is given by

$$
\sigma_{a} \cdot\left(a_{0}, \ldots, a_{4}\right)=\left(a a_{0}, \ldots, a a_{4}\right) \text {, }
$$

where $\sigma_{a}(\zeta)=\zeta^{a}$. This allows us to analyze the $\mathbf{Q}$ structure on $H^{3}(X, \mathbf{C})$. We will also fix the natural orthonormal basis $v_{\mathrm{a}}$ of eigenvectors for $H^{3}(X)$.

To see which characters occur in $H^{1}\left(\Theta_{X}\right)$, it is easiest to use the isomorphism $H^{1}\left(\Theta_{X}\right) \simeq H^{2,1}$ given by cup product with $H^{3,0}$; also observe that elements of $H^{1}\left(\Theta_{X}\right)$ can be identified with quintics $G$ modulo the Jacobian ideal $J=$ $\left(x_{0}^{4}, \ldots, x_{4}^{4}\right)$ of $X$. The element of $H^{1}\left(\Theta_{X}\right)$ corresponding to the first order deformation $F+t G$, where $G=\prod_{i} x_{i}^{a_{i}}$ has character $\left(a_{0}, \ldots, a_{4}\right)$, unless $G \in$ $J$. The cup product mappings $H^{1}\left(\Theta_{X}\right) \otimes H^{p, q} \rightarrow H^{p-1, q+1}$ are $G$-equivariant.

Let $V_{\max } \subset H^{2,1} \oplus H^{1,2}$ be the maximal sub-Hodge structure of $H^{3}(X)$. Then $\operatorname{dim}(V)=200$, and $V$ is spanned by all eigenspaces except $\{(i, i, i$, $i, i)\}$. $V$ is the direct sum of 50 irreducible 4-dimensional Hodge structures. One such is the Hodge structure $V_{a}$ spanned by

$$
\{(1,1,3, a,-a),(2,2,1,2 a,-2 a),
$$

$$
(3,3,4,3 a,-3 a),(4,4,2,4 a,-4 a)\}
$$

for any $1 \leq a \leq 4$. The first two characters span $V^{2,1}$; the latter two span $V^{1,2}$. Other Hodge structures may be obtained from this one by permuting the factors. Actually, we will be using a slightly different decomposition of $V_{\max }$ later.

Now we identify the image of the Abel-Jacobi mapping associated to the cones of lines (compare with [S2, $\S 4]$ ). Let $C=C_{i j r}$ be the cone of lines forming the 
hyperplane section $x_{i}+\zeta^{r} x_{j}=0 . C$ is a cone over the Fermat plane quintic curve $x_{k}^{5}+x_{l}^{5}+x_{m}^{5}=0$, where $\{i, j, k, l, m\}=\{0, \ldots, 4\}$. The Hodge structure on $H^{1}(C)$ can also be described by Ogus' analysis; it corresponds to the characters

$$
\{(1,1,3),(2,2,1),(3,3,4),(4,4,2) \text { plus permutations }\}
$$

of the group $\mathbf{Z}_{5}^{3} / \Delta$. It is clear by looking at characters that, for $C=C_{01 r}$, the image of $H^{1}(C) \rightarrow H^{3}(X)$ is contained in the span of the eigenspaces

$\{(a,-a, 1,1,3),(2 a,-2 a, 2,2,1)$,

$$
(3 a,-3 a, 3,3,4),(4 a,-4 a, 4,4,2) \text { for all } 1 \leq a \leq 4,
$$

plus permutations of the last three coordinates\}

A more careful analysis shows that the image is spanned by the vectors

$$
v_{\left(1,4, a_{0}, a_{1}, a_{2}\right)}-\zeta^{r} v_{\left(2,3, a_{0}, a_{1}, a_{2}\right)}+\zeta^{2 r} v_{\left(3,2, a_{0}, a_{1}, a_{2}\right)}-\zeta^{3 r} v_{\left(4,1, a_{0}, a_{1}, a_{2}\right)}
$$

where $\left(a_{0}, a_{1}, a_{2}\right) \in\{(1,1,3),(2,2,1),(3,3,4),(4,4,2)$ plus permutations $\}$. For future reference, we will denote this Hodge structure by $V_{01 r}$. This 12-dimensional Hodge structure is reducible, reflecting the reducibility of the Hodge structure on $H^{1}(C)$; in fact, one can find three different fixed point free $\mathbf{Z}_{5}$ actions on $C$ (so that the quotients have genus 2, by RiemannHurwitz) which decompose the Hodge structure into irreducibles. These 4dimensional Hodge structures are the Abel-Jacobi images associated to the family of reducible degree 5 curves made up of the 5 lines of the cone intersecting $x_{3}=x_{4}=0$ in the orbits of the corresponding $\mathbf{Z}_{5}$ action on $C$.

From this description it is clear that $\phi^{*}=$ codifferential of the Abel-Jacobi map sends an eigenvector $v_{\left(a_{0}, a_{1}, a_{2}, a_{3}, a_{4}\right)} \in H^{2,1}(X)$ to the eigenvector

$$
v_{\left(a_{2}, a_{3}, a_{4}\right)} \in H^{1,0}(C)=H^{0}\left(K_{C}\right) \text {. }
$$

On the other hand, if $G=\prod_{i} x_{i}^{a_{i}} \in H^{1}\left(\Theta_{X}\right)$, then $\psi(G)=Q(a, b, c)=$ $a^{a_{2}} b^{a_{3}} c^{a_{4}}$, if $a_{2}+a_{3}+a_{4}=2$, and $\psi(G)=0$ otherwise. Recalling the shift in degrees that we have passing from $H^{2,1}(X)$ to $H^{1}\left(\Theta_{X}\right)$, we conclude that $\psi=\phi^{*}$.

Remark. We have seen that the lines that deform (with monodromy) in the direction of $G$ are singled out among all the lines in the family by the zeroes of $\psi(G)$. In the quintic Fermat case, the map $\psi$ has a nice geometric interpretation as the codifferential of the Abel-Jacobi mapping. One could hope for a general statement of this sort: 
Problem 2.5. Let $X$ be a threefold with trivial canonical bundle, and let

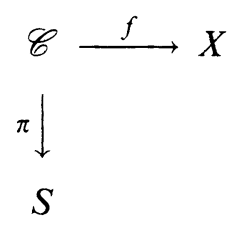

be a family of (rational) curves on $X$ parametrized by a smooth $S$. There is an Abel-Jacobi mapping $\phi: \operatorname{Alb}(S) \rightarrow J(X)$. Then, if $\rho \in H^{1}\left(\Theta_{X}\right)$, the curves that deform (with multiplicity) in the direction of $\rho$ are precisely the curves $\mathscr{C}_{s}$ for which $\phi^{*}(\rho)(s)=0$.

A special case is when $S$ is rational. In this case 2.5 says that the entire family deforms in all directions. Obvious examples are families of complete intersections on $X$. Another example is $X=$ Weierstrass fibration over a rational surface $S$. In this case, identifying $S$ with a section, we have $N_{S / X}=$ $K_{S}$, and hence $H^{1}\left(N_{S / X}\right)=0$. So by Kodaira's theorem [Kod], $S$ deforms with $X$ in all directions.

\section{Deformation theory OF Hodge StRUCtURES}

Let $X$ be a smooth threefold, with associated Hodge structure $H^{3}=H^{3}(X)$ $=\bigoplus H^{p, q}$.

Definition 3.1. An abelian sub-Hodge structure of $H^{3}$ is a sub-Hodge structure contained in $H^{2,1} \oplus H^{1,2}$.

More explicitly, $V$ is an abelian sub-Hodge structure if there is a rational vector space $V_{\mathbf{Q}}$ with $V=V_{\mathbf{Q}} \otimes \mathbf{C}$, such that if $V^{p, q}=V \cap H^{p, q}$, then $V=V^{2,1} \oplus V^{1,2}$. The terminology is chosen because $V$ induces an abelian subvariety of the intermediate Jacobian $J(X)$.

Suppose that the Kuranishi space $\mathscr{M}$ of $X$ is smooth, and isomorphic to a polydisc in $H^{1}\left(\Theta_{X}\right)$, e.g. if $H^{2}\left(\Theta_{X}\right)=0[\mathrm{KNS}]$, or if $K_{X} \equiv 0[\mathrm{~T}$, Theorem 1]. For $p \in \mathscr{M}$, let $X_{p}$ be the corresponding threefold. Then we can think of $H^{3}\left(X_{p}\right)$ as the fixed vector space $H^{3}$ with a varying Hodge structure depending on $p$ [Gr2]. If no $X_{p}$ is specified, it is assumed that the Hodge structure considered is the Hodge structure associated to $X$.

Let $V$ as above be an abelian sub-Hodge structure of $H^{3}$. Let

$$
Z_{V}=\left\{p \in \mathscr{M} \mid V \subset H^{3}\left(X_{p}\right) \text { is an abelian sub-Hodge structure }\right\}
$$

Recall that there are natural cup product mappings

$$
H^{1}\left(\Theta_{X}\right) \otimes H^{p, q} \rightarrow H^{p-1, q+1} .
$$


Proposition 3.2. The tangent space to $Z_{V}$ at $X$ is the set of all $\rho \in H^{1}\left(\Theta_{X}\right)$ such that

(i) $\rho \cdot V^{2,1} \subset V$, and

(ii) $\rho \cdot H^{3,0} \perp V$.

Proof. Let $\rho \in T\left(Z_{V}\right)$. Condition (i) says that $V$ remains a sub-Hodge structure to first order. To see this, let $V_{p}^{2,1}=V \cap H^{2,1}\left(X_{p}\right)$, and similarly define $V_{p}^{1,2}$. Then $p \in Z_{V}$ if and only if $V=V_{p}^{2,1} \oplus V_{p}^{1,2}$. Since $V_{p}^{1,2}$ is the complex conjugate of $V_{p}^{2,1}$, this happens if and only if $\operatorname{dim} V_{p}^{2,1}=\frac{1}{2} \operatorname{dim} V$. In other words, $V_{p}^{2,1} \in \operatorname{Grass}\left(\frac{1}{2} \operatorname{dim} V, V\right)$. Condition (i) follows from the well-known description of the tangent space to a Grassmannian.

Condition (ii) says that $V$ remains abelian to first order, since being abelian is equivalent to being orthogonal to $H^{3,0}$.

It is easy to see that these necessary conditions are also sufficient. Q.E.D.

Let $X$ be a projective threefold with trivial canonical bundle. Then, as we remarked earlier, the Kuranishi space $\mathscr{M}$ for $X$ is an open set in the vector space $H^{1}\left(\Theta_{X}\right)$. Let $V$ be an abelian sub-Hodge structure of $H^{3}(X)$.

Let $D=\mathbf{C}[\varepsilon] / \varepsilon^{2}$ be the ring of dual numbers, and for $\rho \in H^{1}\left(\Theta_{X}\right)$, let $X_{\rho} \rightarrow$ Spec $D$ be the corresponding first order deformation.

We are now ready to state an infinitesimal version of the generalized Hodge conjecture (IGHC).

Conjecture 3.3. (IGHC) Suppose $\rho \in H^{1}\left(\Theta_{X}\right)$ is a first order deformation for which $V$ is contained in an abelian sub-Hodge structure to first order (that is, $\rho \in T\left(Z_{V}^{\prime}\right)$ for some $V^{\prime}$ containing $\left.V\right)$. Then there exists a family of curves $\mathscr{C} \rightarrow C$ on $X$ whose Abel-Jacobi image is $V$, which deforms to first order.

More precisely, there is a diagram

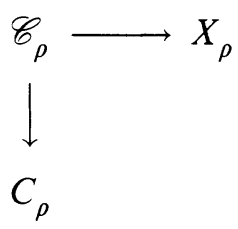

over Spec $D$, with the special fiber of the family $\mathscr{C}_{\rho} \rightarrow C_{\rho}$ a multiple of the family $\mathscr{C} \rightarrow C$.

Remarks. 1. The reason for the need for the multiplicity will become apparent later in analyzing this question for the Fermat quintic threefold.

2. Note that IGHC follows from Problem 2.5: from condition (ii) of Proposition 3.2 , one sees that $\phi^{*}(\rho) \equiv 0 \in H^{0}\left(K_{C}\right)$.

We now specialize to the case of the Fermat quintic threefold. Recall the description of the abelian sub-Hodge structures given above. 
Lemma 3.4. IGHC is true for $V_{\max }$.

Lemma 3.5. IGHC is true for the Abel-Jacobi image of a single cone of lines.

Proposition 3.6. If $V$ is the sum of any number of the Hodge structures $V_{i j r}$, then IGHC holds for $V$.

Proof of 3.4. If $\rho \in T\left(Z_{V}^{\prime}\right)$ for some $V^{\prime}$ containing $V$, then $\rho \cdot H^{3,0} \perp$ $V_{\max }$. Since $H^{3,0}$ consists of the single eigenspace $(1,1,1,1,1)$, we see that $\rho$ must be in the span of the eigenspaces with characters $\left\{\left(a_{0}, \ldots, a_{4}\right) \mid\right.$ $\left.\left(a_{0}+1, \ldots, a_{4}+1\right) \notin V\right\}$. This forces $\rho$ to have weight $(1,1,1,1,1)$. This corresponds to the first order deformation $F+t G=0$, where $G=\prod_{i} x_{i}$.

The proof concludes by the criterion of $\S 2$. The coefficient of $u^{3} v^{2}$ in $G$ is identically 0 for any cone. Thus all cones deform to first order. Q.E.D.

Remark. We have shown here that in the pencil $F+t \prod_{i} x_{i}$, all cones deform to first order. This should be compared to an interesting computation done recently by B. Van Geemen (private communication), who has shown that all the threefolds in this pencil have at least 5000 lines, and hence infinitely many. This raises the interesting question of describing the incidence correspondence of lines on the threefolds in this pencil. In particular, do the cones of lines on the Fermat deform to all orders? ${ }^{1}$

Proof of 3.5. By symmetry, we may as well assume that the cone in question is $C_{010}$, with corresponding Hodge structure $V_{010}$. As in the proof of the previous Proposition, we consider first order deformations $\rho$ for which $\rho$. $H^{3,0} \perp V_{010}$. Writing the first order deformation as $F+t G$, and writing $G=\sum_{\alpha} f_{\alpha}\left(x_{0}, x_{1}\right) g_{\alpha}\left(x_{2}, x_{3}, x_{4}\right)$ with $f_{\alpha}$ homogeneous and $g_{\alpha}$ ranging over distinct monomials, then the condition is easily seen to be that for all $\alpha$ with $\operatorname{deg} f_{\alpha}=3$, then $f_{\alpha}$ is of the form $c_{0} x_{0}^{3}+c_{1} x_{0}^{2} x_{1}+c_{2} x_{0} x_{1}^{2}+c_{3} x_{1}^{3}$, with $c_{0}-$ $c_{1}+c_{2}-c_{3}=0$ (coming from the explicit description of $V_{010}$ ). In other words, $f_{\alpha}$ is divisible by $x_{0}+x_{1}$. Since $x_{0}+x_{1}$ is identically 0 on the cone $C_{010}$, this shows the coefficient of $u^{3} v^{2}$ in the restriction of $G$ to the cone is identically 0 , hence the cone deforms to first order. Q.E.D.

Proof of 3.6. If $\rho \cdot H^{3,0} \perp V$, then $\rho \cdot H^{3,0} \perp V_{i j r}$ for all the involved $V_{i j r}$. By the proof of the previous proposition, all of the lines in the cones $C_{i j r}$ deform to first order. Q.E.D.

To complete the discussion of IGHC for the Fermat quintic threefold, we remark that an easy computation shows that if $V$ is one of the 4-dimensional Hodge structures described above, the cones of lines do not deform in all directions in $T\left(Z_{V}\right)$. This is consistent with our previous examples, since the Abel-Jacobi image of the entire cone is too big. However, this certainly does

${ }^{1}$ Added in proof. The authors have described the incidence correspondence and answered this question affirmatively in a recent preprint. 
not violate IGHC, and we feel that this would be a worthwhile investigation. While we cannot yet prove it, we expect that IGHC can be proven using the genus two family of reducible degree 5 curves above.

\section{REFERENCES}

[Al] A. Albano, Infinite generation of the Griffiths group: a local proof, Thesis, University of Utah, 1986.

[Ar] M. Artin, On the solution of analytic equations, Invent. Math. 5 (1968), 277-291.

[Cl1] C. H. Clemens, Homological modulo algebraic equivalence is not finitely generated, Publ. Math. I.H.E.S. 58 (1983), 231-250.

[C12] — $\mathscr{D}$-modules and subcanonical deformations, Preprint, University of Utah, 1988.

[F] W. Fulton, Intersection theory, Springer-Verlag, Berlin, Heidelberg, and New York, 1984.

[Gr1] P. A. Griffiths, On the periods of certain rational integrals. I, II, Ann. of Math. 90 (1969), 460-541.

[Gr2] , Periods of integrals on algebraic manifolds. I, II, Amer. J. Math. 90 (1968), 568-626, 805-865.

[H] J. Harris, Galois group of enumerative problems, Duke Math. J. 46 (1979), 685-724.

[K1] S. Katz, On the finiteness of rational curves on quintic threefolds, Compositio Math. 60 (1986), 151-162.

[K2] , Degenerations of quintic threefolds and their lines, Duke Math. J. 50 (1983), 11271135.

[K3] _ Tangents to a multiple plane curve, Pacific J. Math. 124 (1986), 321-331.

[KNS] K. Kodaira, L. Nirenberg, and D.C. Spencer, On the existence of deformations of complex analytic structures, Ann. of Math. 68 (1958), 450-459.

[Kod] K. Kodaira, On stability of compact submanifolds of complex manifolds, Amer. J. Math. 85 (1963), 79-94.

[O] A. Ogus, Griffiths transversality and crystalline cohomology, Ann. of Math. 108 (1978), 395-419.

[Ra] Z. Ran, Cycles on Fermat hypersurfaces, Compositio Math. 42 (1980/81), 121-142.

[Re] M. Reid, The moduli space of threefolds with trivial canonical bundle may nevertheless be irreducible, Math. Ann. 278 (1987), 329-334.

[S1] T. Shioda, The Hodge conjecture for Fermat varieties, Math. Ann. 245 (1979), 175-184.

[S2] _ Algebraic cycles on hypersurfaces in $\mathbf{P}^{n}$, Adv. Stud. in Pure Math., vol. 10, NorthHolland, Amsterdam and New York, pp. 717-732.

[T] G. Tian, Smoothness of the universal deformation space of compact Calabi-Yau manifolds and its Petersson-Weil metric, Mathematical Aspects of String Theory (S. T. Yau, ed.), World Scientific, 1987.

Dipartimento di Matematica, Università di Torino, Via Carlo Alberto 10, 10123 TORINO, ITALY 74078

Department of Mathematics, Oklahoma State University, Stillwater, Oklahoma 